\title{
Towards Attosecond High-Energy Electron Bunches: Controlling Self-Injection in Laser-Wakefield Accelerators Through Plasma-Density Modulation
}

\author{
M. P. Tooley, ${ }^{1}$ B. Ersfeld, ${ }^{1}$ S. R. Yoffe, ${ }^{1}$ A. Noble, ${ }^{1}$ E. Brunetti,${ }^{1}$ Z. M. Sheng, ${ }^{1,2,3}$ M. R. Islam, ${ }^{1}$ and D. A. Jaroszynski ${ }^{1}{ }^{1,}$ \\ ${ }^{1}$ Department of Physics, SUPA and University of Strathclyde, Glasgow G4 ONG, United Kingdom \\ ${ }^{2}$ Laboratory for Laser Plasmas and Department of Physics and Astronomy, Shanghai 200240, China \\ ${ }^{3}$ Collaborative Innovation Center of IFSA, Shanghai Jiao Tong University, Shanghai 200240, China
}

(Received 30 August 2016; published 26 July 2017)

\begin{abstract}
Self-injection in a laser-plasma wakefield accelerator is usually achieved by increasing the laser intensity until the threshold for injection is exceeded. Alternatively, the velocity of the bubble accelerating structure can be controlled using plasma density ramps, reducing the electron velocity required for injection. We present a model describing self-injection in the short-bunch regime for arbitrary changes in the plasma density. We derive the threshold condition for injection due to a plasma density gradient, which is confirmed using particle-in-cell simulations that demonstrate injection of subfemtosecond bunches. It is shown that the bunch charge, bunch length, and separation of bunches in a bunch train can be controlled by tailoring the plasma density profile.
\end{abstract}

DOI: 10.1103/PhysRevLett.119.044801

Considerable research effort is currently focused on the development of laser-plasma wakefield accelerators (LWFA) as sources of high-quality, ultrashort electron bunches [1-5] and as potential drivers of free-electron lasers (FELs) [6,7] and tabletop plasma-based light sources [8-13]. Such accelerators promise a new generation of compact, affordable devices with wide-ranging applications in academia, industry, and healthcare. Of particular interest is the production of ultrashort bunches to drive attosecond $\mathrm{x}$-ray sources for ultrafast studies of physical and biological processes.

Plasma waves driven by an intense laser pulse are capable of supporting accelerating fields many orders of magnitude in excess of those possible in conventional accelerators. The strongest accelerating fields are obtained in the "bubble" regime, characterized by a region of complete electron evacuation behind the driving laser, surrounded by a dense electron sheath, producing strong electric fields (in the hundreds of $\mathrm{GV} / \mathrm{m}$ ) [14]. This structure propagates through the plasma with approximately the laser group velocity, and electrons must enter into and copropagate with it to be accelerated. This is most conveniently achieved by "self-injection," where sheath electrons acquire velocities exceeding that of the bubble structure, allowing them to enter the accelerating field region. This avoids the additional complexity of externally injecting electrons.

Self-injection is usually produced by increasing the laser intensity above a certain threshold; however, this does not

Published by the American Physical Society under the terms of the Creative Commons Attribution 4.0 International license. Further distribution of this work must maintain attribution to the author(s) and the published article's title, journal citation, and DOI. provide a means for controlling the bunch properties. While a complete theory of self-injection in the LWFA is currently lacking, several models describing important aspects of the process have been proposed. Initial analyses considered a nonevolving plasma wave [15-17], but predict higher intensity and density requirements for injection to take place than those observed experimentally [18]. More recently, models including an evolving bubble size $[19,20]$ or potential [21] have been developed, which provide more realistic estimates. Furthermore, techniques to control injection have also been proposed, using transverse magnetic fields [22] or further ionization of the plasma [23]. Another approach, which has been demonstrated experimentally $[24,25]$ and numerically [26], is to exploit the expansion of the bubble on a density down-ramp when using a laser and plasma combination that would otherwise preclude injection. A theoretical treatment of this process considers Langmuir wave breaking [27] for a constant bubble velocity, but this leads to overestimation of the required density gradient. The manipulation of injection and bunch properties remains an outstanding challenge, particularly for the production of ultrashort bunches [21,28].

In this Letter, we present a model describing selfinjection of ultrashort bunches towards attosecond duration. We show how arbitrary plasma density gradients give rise to changes in the phase velocity of the bubble that can lead to injection. We find a threshold condition that enables determination and control of the injected bunch, and show that subfemtosecond bunches are achievable using relatively modest laser parameters. Results from particle-in-cell (PIC) simulations demonstrate the injection of such bunches in excellent agreement with our model.

Our model describes injection of plasma electrons that propagate along the sheath before entering the rear of the bubble. Such electrons run ahead of the wave phase and so 
satisfy the definition of wave breaking. An electron is considered to be injected when it has entered the accelerating field; therefore, the zero-crossing point of the axial electric field $E_{z}(z)=0$ is used to mark the rear of the bubble. It is the velocity of this point, which we refer to as the "bubble phase velocity" and denote $\beta_{b}$, that has a key role in determining injection.

Before presenting the analytical model, we demonstrate how density modulations alter the evolution of the bubble phase velocity in 2D PIC simulations using ЕРОCH [29]. The plasma density is $n_{e}(z)=n_{c} \eta^{2}(z)$, with the profile

$\frac{\eta^{2}}{\eta_{0}^{2}}= \begin{cases}\theta(z) z / r & \text { if } z \leq r \\ 1+\theta\left(w-\left|z-z_{i}\right|\right) \alpha \cos ^{2}\left(\pi \frac{z-z_{i}}{2 w}\right) & \text { if } z>r\end{cases}$

where $\theta(z)$ is the Heaviside function, $\eta_{0}^{2}$ is the plateau density fraction of the critical density $n_{c}=\omega^{2} \epsilon_{0} m_{e} / e^{2}$ for laser frequency $\omega, r$ is the length of the linear up-ramp, and $z_{i}$ is the position of a density peak of half-width $w$ and relative amplitude $\alpha$. The density profile does not vary perpendicular to the laser axis. This form of density profile, illustrated in Fig. 1, is achievable using supersonic de Laval gas nozzles [30] or by introducing shocks into the source gas flow [31]. A matched, linearly polarized laser beam with initial peak normalized amplitude $a_{0}=4$ is used to drive the wakefield to minimize laser evolution due to selffocusing [32]. Simulations are performed using a cell size of $40 \mathrm{~nm} \times 200 \mathrm{~nm}$ with 16 particles per cell. The bubble phase velocity extracted from a PIC simulation for $\alpha=0.1$ is shown in Fig. 1, along with the prediction from the model (4) below for the same parameters. In this case injection does not occur.

In order to describe electron self-injection in the bubble regime, the trajectories of individual plasma electrons must be carefully considered. The ponderomotive force of the laser pulse displaces initially stationary plasma electrons, which then oscillate transversely at the betatron frequency $\omega_{\beta}=\omega_{p} / \sqrt{\Gamma_{e}}=2 \pi \eta c /\left(\lambda \sqrt{\Gamma_{e}}\right)$, where $\omega_{p}=\eta \omega$ is the plasma frequency and $\lambda$ is the laser wavelength. The ratio of the frequencies depends on $\Gamma_{e}=\gamma_{e}$ in $2 \mathrm{D}$, or $\Gamma_{e}=2 \gamma_{e}$

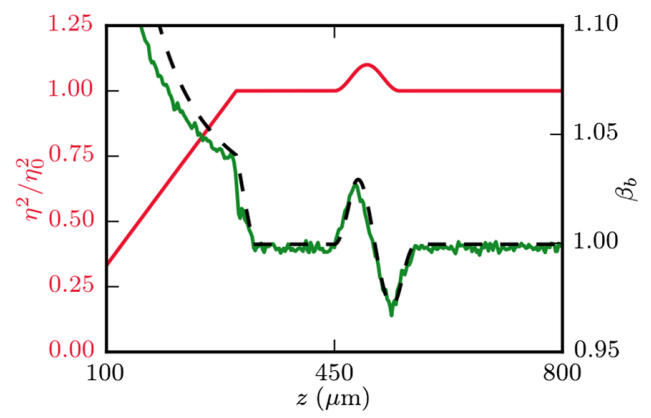

FIG. 1. Bubble phase velocity $\beta_{b}$ from PIC data (green) and calculated using (4) (black dashed), for the density profile (1) (red). Chosen parameters were $a_{0}=4, \lambda=800 \mathrm{~nm}$, and $\eta_{0}^{2}=0.001$. After a linear ramp of $r=300 \mu \mathrm{m}$, a density peak with amplitude $\alpha=0.1$ and width $w=50 \mu \mathrm{m}$ is located at $z_{i}=500 \mu \mathrm{m}$. in $3 \mathrm{D}$, where $\gamma_{e}$ is the electron Lorentz factor. In the ponderomotive approximation, $\gamma_{e} \simeq \sqrt{1+a^{2} / 2}$, where $a=e E / m c \omega$ is the peak normalized field amplitude. An electron initially located close to the laser axis returns to it after half an oscillation period, $\tau=\pi / \omega_{\beta}$. This determines the length of the bubble, since electrons driven from the axis by the laser at time $t_{\ell}$ form the back of the bubble at time $t_{b}=t_{\ell}+\tau(z)$. Differentiating with respect to $z$ gives

$$
\frac{1}{\beta_{b}}=\frac{1}{\beta_{\mathrm{gr}}}+c \frac{d \tau}{d z}
$$

with the laser group and bubble phase velocities defined as $c \beta_{\mathrm{gr}}=\left(d t_{\ell} / d z\right)^{-1}=c \sqrt{1-\eta^{2}}$ and $c \beta_{b}=\left(d t_{b} / d z\right)^{-1}$, respectively. The difference between the bubble phase velocity and the group velocity is determined by the rate of change of the flight time $\tau$ for electrons crossing the laser axis at the back of the bubble (i.e., the rate of change of the bubble length as it propagates). Such relationships between a driver and trailing structure are sometimes termed the "accordion effect" [33,34].

In practice, not all of the electrons start directly on the laser axis. However, a trajectory beginning off axis may be considered as part of an equivalent trajectory which began on axis at an earlier time. In addition, the electrons do not oscillate purely transversely, but rather gain some forward longitudinal momentum. Both of these effects may be approximated by considering electrons crossing the axis at position $z$ to have originated from a range of apparent positions $\tilde{z} \leq z$.

The difference between the bubble phase velocity and the group velocity in (2) depends on the trajectories of all those electrons crossing the axis at $z$. It is therefore necessary to average (denoted $\langle\cdots\rangle$ ) over all possible initial electron positions $z-\lambda_{p}<\tilde{z} \leq z$, where $\lambda_{p}=\lambda / \eta$ is the plasma wavelength, for electrons that can form the back of the bubble,

$$
\frac{1}{\beta_{b}}=\frac{1}{\beta_{\mathrm{gr}}}+c\left\langle\frac{d \tau}{d z}\right\rangle
$$

from which the phase velocity is expressed as

$$
\beta_{b}=\beta_{\mathrm{gr}}\left(1-\frac{\beta_{\mathrm{gr}} \lambda}{2}\left\langle\frac{\eta^{\prime} \sqrt{\Gamma_{e}}}{\eta^{2}}-\frac{\Gamma_{e}^{\prime}}{2 \eta \sqrt{\Gamma_{e}}}\right\rangle\right)^{-1},
$$

where prime denotes differentiation with respect to $z$.

In the case of a constant density plasma and nonevolving $\operatorname{driver}\left(\eta^{\prime}=\Gamma_{e}^{\prime}=0\right)$, the expression reduces to $\beta_{b}=\beta_{\mathrm{gr}}$, as expected. For a positive density gradient $\eta^{\prime}>0$ the bubble length decreases, increasing $\beta_{b}$ despite the reduction in the group velocity $\beta_{\mathrm{gr}}$. Indeed, $\beta_{b}$ can exceed unity for a sufficiently large positive density gradient, completely suppressing electron self-injection as electrons can never enter the bubble [25]. Conversely, with a negative gradient the bubble lengthens, reducing $\beta_{b}$. In this Letter the laser spot size is matched to the plasma density. Otherwise, rapid evolution of the laser pulse can cause the $\Gamma_{e}^{\prime}$ term to 

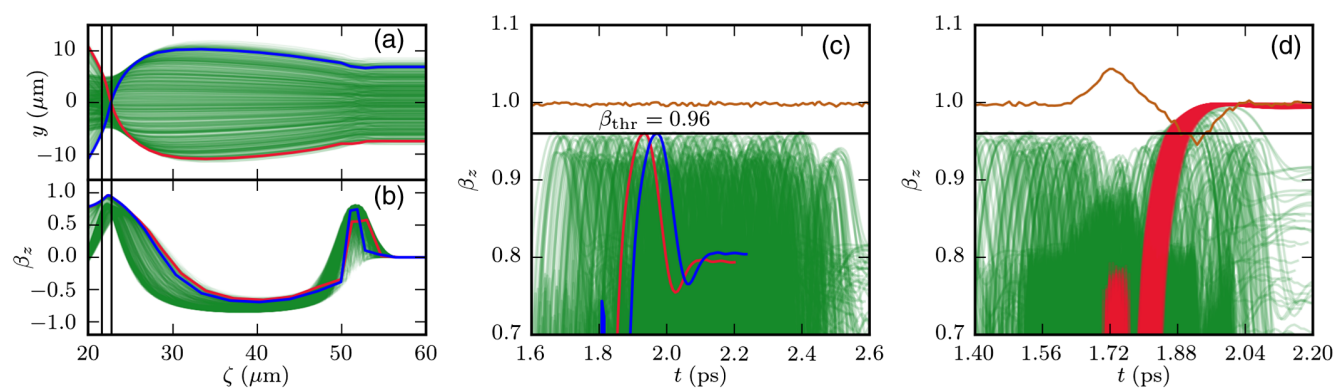

FIG. 2. Trajectories of a random selection of sheath electrons for $\eta_{0}^{2}=0.001$ and $\alpha=0[\alpha=0.15$ for panel (d)] and laser parameters as in Fig. 1. Panel (a) shows the spatial trajectories of electrons in the frame comoving with the bubble, and panel (b) the variation of their longitudinal velocities $\beta_{z}$. Red and blue lines highlight typical trajectories. Panels (c) and (d) show the evolution of the longitudinal electron velocities. In the latter, the injected population is shown in red. Orange lines show the bubble phase velocity, and horizontal black lines denote the threshold velocity.

dominate, making prediction and control of injection far more complex.

Injection occurs when the bubble phase velocity drops below the peak electron velocity, allowing the fastest electrons to penetrate into the bubble. This peak electron velocity is the threshold bubble phase velocity $\beta_{\text {thr }}$. Applying the wave-breaking condition $\beta_{b}<\beta_{\text {thr }}$ to (4) and rearranging yields

$$
\left\langle\frac{\eta^{\prime} \sqrt{\Gamma_{e}}}{\eta^{2}}\right\rangle-\left\langle\frac{\Gamma_{e}^{\prime}}{2 \eta \sqrt{\Gamma_{e}}}\right\rangle<\frac{2}{\lambda}\left(\frac{1}{\beta_{\mathrm{gr}}}-\frac{1}{\beta_{\mathrm{thr}}}\right) .
$$

For cases where the evolution of the driver can be neglected (such as $\gamma_{e}=\gamma_{0}=\sqrt{1+a_{0}^{2} / 2}$ and $\Gamma_{e}=\Gamma_{0}$ for a matched laser) this reduces to

$$
\left\langle\frac{\lambda_{p} \eta^{\prime}}{\eta}\right\rangle<\frac{2}{\sqrt{\Gamma_{0}}}\left(\frac{1}{\beta_{\mathrm{gr}}}-\frac{1}{\beta_{\mathrm{thr}}}\right)
$$

The quantity $\lambda_{p} \eta^{\prime} / \eta$ approximates the relative change in $\eta$ over one plasma wavelength.

The injected bunch length is determined from the interval during which the wake phase velocity $\beta_{b}$ remains below the injection threshold velocity $\beta_{\text {thr }}$. Assuming the profile is such that $\beta_{b}<\beta_{\text {thr }}$ throughout an interval $z_{0}<$ $z<z_{1}$ and $\beta_{b}>\beta_{\text {thr }}$ outside this interval, the positions $z_{0}$ and $z_{1}$ define the start and end points for the injection episode. The injection period is found to be

$$
\Delta t_{\mathrm{inj}}=\frac{1}{c} \int_{z_{0}}^{z_{1}} \frac{1}{\beta_{b}\left(z^{\prime}\right)} d z^{\prime}=\frac{z_{1}-z_{0}}{c \bar{\beta}_{b}}
$$

where $\bar{\beta}_{b}$ is the harmonic mean of $\beta_{b}$ over the injection length. The corresponding bunch length can be predicted by considering the distance, relative to the back of the bubble, traveled by an electron injected at the beginning of the injection period during the interval $\Delta t_{\mathrm{inj}}$. Assuming that the injected electrons are accelerated sufficiently rapidly that their normalized velocity $\beta_{z}$ may be taken to be unity, we may approximate the bunch length $\ell$ as

$$
\ell \simeq \frac{1-\bar{\beta}_{b}}{\bar{\beta}_{b}}\left(z_{1}-z_{0}\right) \simeq \frac{\Delta L}{2 \bar{\gamma}_{b}^{2}},
$$

with $\bar{\gamma}_{b}=\left(1-\bar{\beta}_{b}^{2}\right)^{-1 / 2}$ the Lorentz factor associated with the average velocity and $\Delta L=z_{1}-z_{0}$. It should be noted that the bunch length may evolve due to variations in the acceleration gradient, energy spread, and bunch charge. The same analysis can be extended to the case of two separate bunches injected as a result of two spatially separated density peaks in the plasma. In this case, taking the points $z_{0}, z_{1}$ to be at the two minima of $\beta_{b}$ associated with adjacent density peaks yields the spacing between individual bunches.

To determine $\beta_{\text {thr }}$ we consider the behavior of sheath electrons. Since there is (currently) no complete analytical description of the electron motion in the laser and bubble fields, we determine the threshold value from a simulation of a flat plasma $(\alpha=0)$ that is otherwise identical to Fig. 1. We analyze the behaviour of electrons that receive a large ponderomotive kick $\left(\gamma_{e} \gtrsim 2\right)$, because it is these higherenergy electrons that form the bubble sheath. Figure 2(a) shows the sheath electron trajectories in the frame comoving with the laser pulse $\left(\zeta=z-c \beta_{\mathrm{gr}} t\right)$. The region where sheath electrons cross the laser axis (bracketed by vertical lines) at the bubble rear can be clearly identified, and Fig. 2(b) indicates that the longitudinal velocity $\beta_{z}$ also peaks in this region. Figure 2(c) shows that there exists a

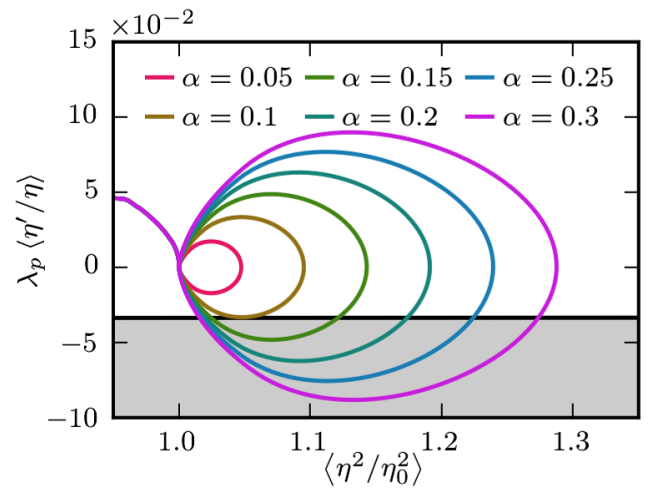

FIG. 3. Variation of the average plasma density gradient for the density profile (1) with $\alpha \in[0.05,0.3]$. The region for which selfinjection can occur, as described by (6), is shaded grey. $\Gamma_{e}=6$ corresponding to $a_{0}=4$. 


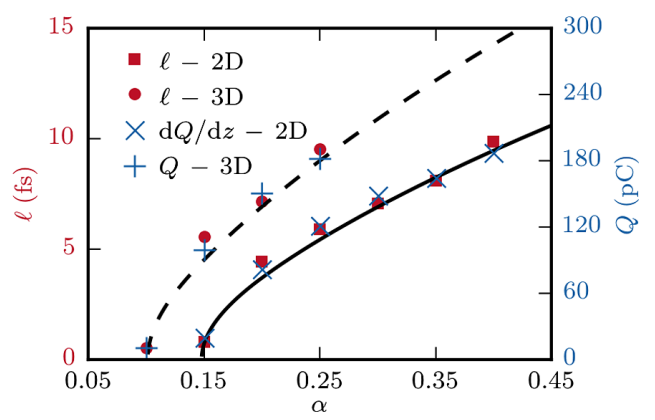

FIG. 4. Injected bunch length (square, circle) and charge (times, plus) for simulations with plasma density parameters as in Fig. 3 for $2 \mathrm{D}$ and $3 \mathrm{D}$ cases. Injected bunch length predicted by the model is shown for comparison with solid (2D) and dashed (3D) lines. The 2D case differs due to the geometric dependence of $\Gamma_{e}$. The $2 \mathrm{D}$ charge density is scaled by a factor of $1.2 \times 10^{-5} \mathrm{~m}$.

continuous flow of sheath electrons at the back of the bubble with a peak velocity $\beta_{\max } \simeq 0.96$. Assuming that this is not significantly altered by small density modulations, $\beta_{\text {thr }}=\beta_{\max }$ is the threshold below which $\beta_{b}$ must drop for electrons to be injected. Figure 2(d) shows the longitudinal electron velocities and the bubble phase velocity in the presence of a plasma density peak sufficient for injection. In addition to the injected electrons, some electrons with velocities exceeding the phase velocity are not injected as their transverse momenta are too large. Although the density perturbation affects electron velocities, we find that the injection criterion assuming unchanged $\beta_{\max }$ gives excellent agreement with simulation results. Therefore, only a single $2 \mathrm{D}$ PIC simulation is needed to provide predictions for arbitrary plasma modulations with a given base density.

Inserting $\beta_{\text {thr }}=\beta_{\max }$ into (6) yields a threshold density gradient for injection that we compare in Fig. 3 to the variation of $\left\langle\lambda_{p} \eta^{\prime} / \eta\right\rangle$ for the profile (1) with $\alpha \in[0.05,0.3]$. For these parameters, we expect injection to occur when the decrease in $\eta$ per plasma wavelength exceeds approximately $4 \%$, which is satisfied by $\alpha \gtrsim 0.10$. The small density gradient required for this stimulated self-injection has implications for situations where injection is not desired; irregularities in the density profile presented by a gas jet or capillary target can cause unwanted or premature self-injection.

We performed a series of simulations using (1) with a single density peak of half-width $w=50 \mu \mathrm{m}$ and varying peak amplitude $\alpha=0.05-0.4$. Injected bunch lengths and charge densities from a parameter scan with 2D and 3D PIC are shown in Fig. 4. As predicted, no injection occurs for density peaks with $\alpha<0.10$ ( 0.15 for $2 \mathrm{D})$, and above this threshold the bunch length increases in good agreement with the model. Close to threshold we find that it is possible to inject subfemtosecond bunches. Duration may be further reduced by increasing the laser intensity such that the electron maximum velocity $\beta_{\text {thr }}$ is larger, leading to an increased value of $\bar{\beta}_{b}$ over the injection period and a greater Lorentz contraction. We note that, for the density profile used, the distance $\Delta L$ initially increases rapidly with $\alpha$. However, above $\alpha \sim 0.3$ this dependence becomes weak, such that the bunch length $\ell$ is mainly determined by the time the bubble takes to travel this distance, captured by the factor $1 /\left(2 \bar{\gamma}_{b}^{2}\right)$ in (8). Bunch charge scales proportionally with bunch length, giving an average beam current of $\sim 15 \mathrm{kA}$. Bunch charge density in 3D for $\alpha=0.10$ and 0.25 are shown in Fig. 5. The smaller $\alpha=0.10$ shown in Fig. 5(a), which is just above threshold, results in a compact bunch with high average charge density. In contrast the density amplitude $\alpha=0.25$ in Fig. 5(b) leads to the injection of a larger total charge (and peak charge density) but a highly asymmetric bunch shape. This is a signature of beam loading, which for large bunch charge (in the hundreds of $\mathrm{pC}$ ) can distort the bubble shape, reducing $\bar{\beta}_{b}$, and causing a lengthening of injected bunches.

In conclusion, we have developed a model for timedependent self-injection in the LWFA operating close to threshold by modifying the bubble phase velocity through variation of plasma density gradients. We have demonstrated the use of this model to control the location, length, and charge of self-injected electron bunches in excellent agreement with PIC simulations. Ultrashort bunches may be obtained using modest laser intensities in a standard LWFA setup. For example, we find that a plateau plasma density $n_{e}=1.74 \times 10^{18} \mathrm{~cm}^{-3}$ with a $10 \%$ density peak of half-width $50 \mu \mathrm{m}$ can be used with a laser $a_{0}=4$ to inject a
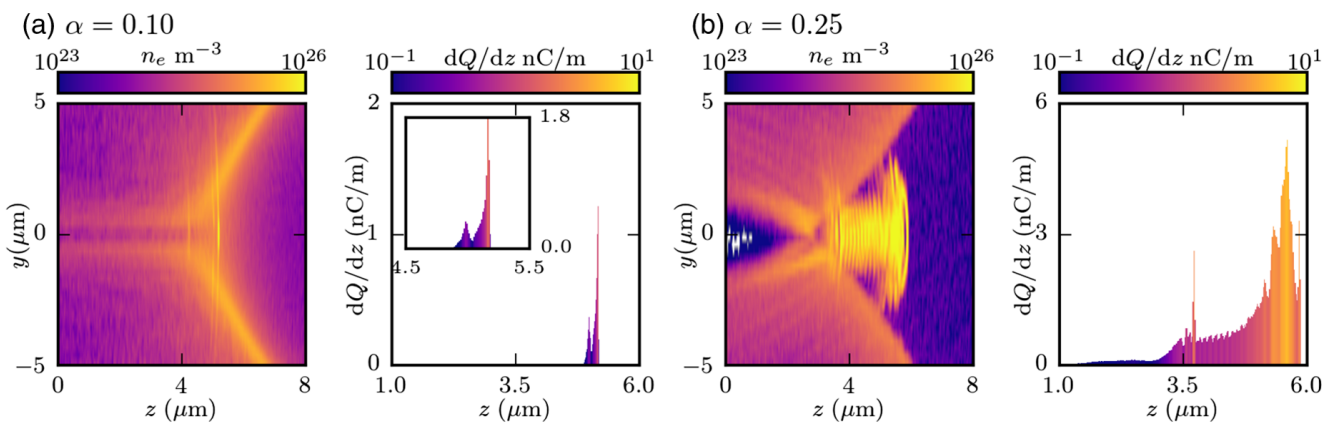

FIG. 5. Comparison of injected bunches from 3D simulations in Fig. 4: (a) $\alpha=0.10$, just exceeding the injection threshold, and (b) $\alpha=0.25$, significantly exceeding the injection threshold. 
bunch with rms duration of 260 attoseconds. This should be compared to recently suggested schemes requiring either complex experimental layout [35] or relatively high laser power [33]. Such control of the bunch parameters has particular significance for FELs and attosecond x-ray sources, for which this method may be used to produce the required ultrashort high-current bunches.

Simulation data associated with the research published here are available by following the link in Ref. [36].

This work was supported by UK Engineering and Physical Sciences Research Council (EPSRC) Grants No. EP/J018171/1, No. EP/J500094/1, and No. EP/ N028694/1, and the EC's H2020 LASERLAB-EUROPE (Grant No. 654148), EuCARD-2 (Grant No. 312453), EuPRAXIA (Grant No. 653782), and a Leverhulme Trust Research Grant. Results were obtained using the EPSRCfunded ARCHIE-WeSt High Performance Computer (www .archie-west.ac.uk), Grant No. EP/K000586/1, and the ARCHER UK National Supercomputing Service. The EPOCH code used in this research was developed under EPSRC Grants No. EP/G054940/1, No. EP/G055165/1, and No. EP/G056803/1. Z. M. S. acknowledges the support of the National Basic Research Program of China (Grant No. 2013CBA01500) and NSFC Grants No. 11421064 and No. 11374210.

*d.a.jaroszynski@strath.ac.uk

[1] S. P. D. Mangles, C. D. Murphy, Z. Najmudin, A. G. R. Thomas, J. L. Collier, A. E. Dangor, E. J. Divall, P. S. Foster, J. G. Gallacher, C. J. Hooker, D. A. Jaroszynski, A. J. Langley, W. B. Mori, P. A. Norreys, F. S. Tsung, R. Viskup, B. R. Walton, and K. Krushelnick, Nature (London) 431, 535 (2004).

[2] C. G. R. Geddes, C. Toth, J. van Tilborg, E. Esarey, C. B. Schroeder, D. Bruhwiler, C. Nieter, J. Cary, and W. P. Leemans, Nature (London) 431, 538 (2004).

[3] J. Faure, Y. Glinec, A. Pukhov, S. Kiselev, S. Gordienko, E. Lefebvre, J.-P. Rousseau, F. Burgy, and V. Malka, Nature (London) 431, 541 (2004).

[4] E. Brunetti, R. P. Shanks, G. G. Manahan, M. R. Islam, B. Ersfeld, M. P. Anania, S. Cipiccia, R. C. Issac, G. Raj, G. Vieux, G. H. Welsh, S. M. Wiggins, and D. A. Jaroszynski, Phys. Rev. Lett. 105, 215007 (2010).

[5] D. A. Jaroszynski, R. Bingham, E. Brunetti, B. Ersfeld, J. Gallacher, B. van der Geer, R. Issac, S. P. Jamison, D. Jones, M. de Loos, A. Lyachev, V. Pavlov, A. Reitsma, Y. Saveliev, G. Vieux, and S. M. Wiggins, Phil. Trans. R. Soc. A 364, 689 (2006).

[6] D. A. Jaroszynski and G. Vieux, AIP Conf. Proc. 647, 902 (2002).

[7] H.-P. Schlenvoigt, K. Haupt, A. Debus, F. Budde, O. Jäckel, S. Pfotenhauer, H. Schwoerer, E. Rohwer, J. G. Gallacher, E. Brunetti, R. P. Shanks, S.M. Wiggins, and D. A. Jaroszynski, Nat. Phys. 4, 130 (2008).

[8] E. Esarey, B. A. Shadwick, P. Catravas, and W. P. Leemans, Phys. Rev. E 65, 056505 (2002).
[9] S. Wang, C. E. Clayton, B. E. Blue, E. S. Dodd, K. A. Marsh, W. B. Mori, C. Joshi, S. Lee, P. Muggli, T. Katsouleas, F. J. Decker, M. J. Hogan, R. H. Iverson, P. Raimondi, D. Walz, R. Siemann, and R. Assmann, Phys. Rev. Lett. 88, 135004 (2002).

[10] A. Rousse, K. T. Phuoc, R. Shah, A. Pukhov, E. Lefebvre, V. Malka, S. Kiselev, F. Burgy, J.-P. Rousseau, D. Umstadter, and D. Hulin, Phys. Rev. Lett. 93, 135005 (2004).

[11] S. Kneip et al., Nat. Phys. 6, 980 (2010).

[12] S. Cipiccia et al., in Advances in X-Ray Free-Electron Lasers Instrumentation III, SPIE Proceedings Vol. 9512 (SPIE-International Society for Optical Engineering, Bellingham, WA, 2015), p. 95121A.

[13] B. Ersfeld, R. Bonifacio, S. Chen, M. R. Islam, P. W. Smorenburg, and D. A. Jaroszynski, New J. Phys. 16, 093025 (2014).

[14] A. Pukhov and J. Meyer-ter Vehn, Appl. Phys. B 74, 355 (2002).

[15] S. V. Bulanov, N. Naumova, F. Pegoraro, and J. Sakai, Phys. Rev. E 58, R5257 (1998).

[16] A. Oguchi, A. Zhidkov, K. Takano, E. Hotta, K. Nemoto, and K. Nakajima, Phys. Plasmas 15, 043102 (2008).

[17] E. Esarey, P. Sprangle, J. Krall, and A. Ting, IEEE Trans. Plasma Sci. 24, 252 (1996).

[18] C. Benedetti, C. B. Schroeder, E. Esarey, F. Rossi, and W. P. Leemans, Phys. Plasmas 20, 103108 (2013).

[19] S. Y. Kalmykov, S. A. Yi, V. Khudik, and G. Shvets, Phys. Rev. Lett. 103, 135004 (2009).

[20] X. F. Li, Y. J. Gu, Q. Yu, S. Huang, F. Zhang, Q. Kong, and S. Kawata, Phys. Plasmas 21, 073109 (2014).

[21] M. R. Islam, E. Brunetti, R. P. Shanks, B. Ersfeld, R. C. Issac, S. Cipiccia, M. P. Anania, G. H. Welsh, S. M. Wiggins, A. Noble, R. A. Cairns, G. Raj, and D. A. Jaroszynski, New J. Phys. 17, 093033 (2015).

[22] J. Vieira, S. F. Martins, V. B. Pathak, R. A. Fonseca, W. B. Mori, and L. O. Silva, Phys. Rev. Lett. 106, 225001 (2011).

[23] C. McGuffey, A. G. R. Thomas, W. Schumaker, T. Matsuoka, V. Chvykov, F. J. Dollar, G. Kalintchenko, V. Yanovsky, A. Maksimchuk, K. Krushelnick, V. Y. Bychenkov, I. V. Glazyrin, and A. V. Karpeev, Phys. Rev. Lett. 104, 025004 (2010).

[24] C. G. R. Geddes, K. Nakamura, G. R. Plateau, C. Toth, E. Cormier-Michel, E. Esarey, C. B. Schroeder, J. R. Cary, and W. P. Leemans, Phys. Rev. Lett. 100, 215004 (2008).

[25] A. J. Gonsalves, K. Nakamura, C. Lin, D. Panasenko, S. Shiraishi, T. Sokollik, C. Benedetti, C. B. Schroeder, C. G. R. Geddes, J. van Tilborg, J. Osterhoff, E. Esarey, C. Toth, and W. P. Leemans, Nat. Phys. 7, 862 (2011).

[26] T. Ohkubo, A. Zhidkov, T. Hosokai, K. Kinoshita, and M. Uesaka, Phys. Plasmas 13, 033110 (2006).

[27] A. V. Brantov, T. Z. Esirkepov, M. Kando, H. Kotaki, V. Y. Bychenkov, and S. V. Bulanov, Phys. Plasmas 15, 073111 (2008).

[28] O. Lundh, J. Lim, C. Rechatin, L. Ammoura, A. Ben-Ismail, X. Davoine, G. Gallot, J.-P. Goddet, E. Lefebvre, V. Malka, and J. Faure, Nat. Phys. 7, 219 (2011).

[29] T. D. Arber, K. Bennett, C. S. Brady, A. Lawrence-Douglas, M. G. Ramsay, N. J. Sircombe, P. Gillies, R. G. Evans, H. Schmitz, A. R. Bell, and C. P. Ridgers, Plasma Phys. Controlled Fusion 57, 113001 (2015). 
[30] K. Schmid and L. Veisz, Rev. Sci. Instrum. 83, 053304 (2012).

[31] K. Schmid, A. Buck, C. M. S. Sears, J. M. Mikhailova, R. Tautz, D. Herrmann, M. Geissler, F. Krausz, and L. Veisz, Phys. Rev. ST Accel. Beams 13, 091301 (2010).

[32] W. Lu, C. Huang, M. Zhou, M. Tzoufras, F. S. Tsung, W. B. Mori, and T. Katsouleas, Phys. Plasmas 13, 056709 (2006).
[33] F. Y. Li, Z. M. Sheng, Y. Liu, J. Meyer-ter-Vehn, W. B. Mori, W. Lu, and J. Zhang, Phys. Rev. Lett. 110, 135002 (2013).

[34] T. Katsouleas, Phys. Rev. A 33, 2056 (1986).

[35] J. Zhu, R. W. Assmann, M. Dohlus, U. Dorda, and B. Marchetti, Phys. Rev. ST Accel. Beams 19, 054401 (2016).

[36] See http://dx.doi.org/10.15129/4b58d502-415f-4064-b3b7$5966 \mathrm{dbc0e679.}$ 